\section{Dement Geriatr Cogn Disord Extra 2011;1:381-392}

DOI: 10.1159/000333368

๔ 2011 S. Karger AG, Basel

Published online: November 9, 2011

www.karger.com/dee

\title{
Brain Alterations and Mini-Mental State Examination in Patients with Progressive Supranuclear Palsy: Voxel-Based Investigations Using ${ }^{18}$ F-Fluorodeoxyglucose Positron Emission Tomography and Magnetic Resonance Imaging
}

\author{
Ryuichi Takahashi $^{\mathrm{a}}$ Kazunari Ishii ${ }^{\text {c, }}{ }^{\text {Tatsuya Kakigi }}{ }^{\mathrm{b}}$ \\ Kazumasa Yokoyama a Etsuro Morie, ${ }^{f}$ Takamichi Murakamic
}

Departments of a Neurology and ${ }^{b}$ Cognitive Disorders, Hyogo Prefectural Rehabilitation Hospital at Nishi-Harima, Tatsuno, 'Department of Radiology, Kinki University Faculty of Medicine, Osakasayama, ${ }^{\mathrm{d}}$ Department of Radiology and Nuclear Medicine, and ${ }^{\mathrm{e}}$ Institute for Aging Brain and Cognitive Disorders, Hyogo Brain and Heart Center, Himeji, and ${ }^{\mathrm{f} B e h a v i o r a l ~ N e u r o l o g y}$ and Cognitive Neuroscience, Tohoku University Graduate School of Medicine, Sendai, Japan

\section{Key Words}

DARTEL · Gray matter $\cdot$ Hypometabolism $\cdot$ Magnetic resonance imaging $\cdot$ Progressive supranuclear palsy $\cdot$ Voxel-based morphometry $\cdot$ White matter

\begin{abstract}
Background/Aims: The aim of this study was to compare differences in morphological and functional changes in brain regions in individual patients with progressive supranuclear palsy (PSP) and correlate their Mini-Mental State Examination (MMSE) score with anatomy and function using magnetic resonance imaging (MRI) and ${ }^{18} \mathrm{~F}$-fluorodeoxyglucose positron emission tomography (FDG-PET). Methods: Sixteen PSP patients and 20 age-matched healthy volunteers underwent FDG-PET and 3-dimensional MRI. Gray matter, white matter and metabolic activity were compared between patients and normal controls. In addition, possible correlations between the MMSE score and brain function/anatomy were examined. Results: The PSP group had reduced cerebral glucose metabolism, and lower gray and white matter volumes in the frontal lobes and midbrain compared with normal controls. In PSP subjects, the metabolic changes observed in the PET scans were greater than the loss in gray and white matter ob-
\end{abstract}


served in the MRI scans. The MMSE scores were positively correlated with volume and FDG uptake in the frontal lobe. Conclusion: FDG-PET is a more effective tool in the diagnosis of PSP than MRI. Atrophy and hypometabolism in the frontal lobe are as important as in the basal midbrain for differentiating PSP patients who primarily exhibit cognitive dysfunction from normal controls.

Copyright $\odot 2011$ S. Karger AG, Basel

\section{Introduction}

Progressive supranuclear palsy (PSP) is the second most common cause of parkinsonism $[1,2]$. Common symptoms of PSP include parkinsonism with progressive vertical gaze palsy, postural instability with falls, akinesia, and cognitive impairment [3, 4]. Further, PSP causes neuronal degeneration in the brainstem, including in the substantia nigra, tegmentum, and superior colliculus [3]; tau pathology that accompanies tuft-shaped astrocytes [5] has also been observed in the superior and middle frontal gyri in PSP [6].

Earlier magnetic resonance imaging (MRI) studies have primarily used regions of interest analyses to examine brain atrophy [7-11]. However, the results of such analyses depend largely on the arbitrary locus and size that examiners select retrospectively. Also, this method is time-consuming and only measures relatively large areas, potentially overlooking finer differences.

Voxel-based analysis using statistical parametric mapping (SPM) was developed to examine focal differences in brain anatomy. This method can eliminate the effects of differences in size, and voxel-by-voxel comparison allows one to perform a prospective statistical analysis of differences in gray matter loss and changes in metabolism.

Using voxel-based morphometry (VBM) with MRI $[12,13]$ and voxel-based positron emission tomography (PET) analysis [14-18] of PSP cases, reductions in gray matter and metabolic activity have been observed primarily in the frontal lobe and basal midbrain.

However, patients in previous PET and MRI studies have differed with regard to the clinical stage of the disease. Thus, a comparison between the metabolic changes and decreases in gray matter in patients is difficult to make. The strength of the present study is a direct comparison of fluorodeoxyglucose (FDG) and VBM in the same PSP subjects.

Based on the success of large multicenter Alzheimer disease neuroimaging studies (e.g. $\mathrm{ADNI}$ and $\mathrm{AIBL}$ ), there is increasing interest in neuroimaging measures as secondary or surrogate markers of outcome for therapy trials on neurodegenerative diseases. The modality that can diagnose PSP in its early stages more adequately remains to be identified. The primary aim of this study was to compare the distribution of glucose hypometabolism and atrophy simultaneously in the same group of patients.

PSP is a major cause of dementia. The cognitive disturbances that occur in PSP are referred to as subcortical dementia $[19,20]$. PSP patients demonstrate significant deficits in recall and moderate forgetfulness, despite intact short-term and implicit perceptual memory [19]. PSP is also accompanied by memory loss and more common deficits, including executive dysfunction, decreased working memory, psychomotor slowing, non-fluent aphasia, and, at times, profound behavioral changes that mimic frontotemporal dementia $[21,22]$.

Despite the clinical significance of dementia, no study has examined the brain structures that are affected during cognitive impairment in patients with PSP. In this study, we selected PSP patients with relatively mild cognitive impairment and examined the changes in the associated brain regions. 
Table 1. Characteristics and symptoms of the 16 PSP patients

\begin{tabular}{l|l}
\hline Dement Geriatr Cogn Disord Extra 2011;1:381-392 \\
\hline \begin{tabular}{l|l} 
DOI: 10.1159/000333368 & $\begin{array}{l}\text { @ } 2011 \text { S. Karger AG, Basel } \\
\text { www.karger.com/dee }\end{array}$ \\
Published online: November 9, 2011 &
\end{tabular}
\end{tabular}

Takahashi et al.: Brain Alterations and MMSE in Progressive Supranuclear Palsy

\begin{tabular}{lc}
\hline Females/males & $5 / 11$ \\
Mean age, years & $64.6 \pm 6.4$ \\
Mean MMSE score & $21.0 \pm 4.4$ \\
Lim rigidity, n & $11(69 \%)$ \\
Postural instability, $\mathrm{n}$ & $16(100 \%)$ \\
Apraxia, n & $0(0 \%)$ \\
Cortical sensory loss, $\mathrm{n}$ & $0(0 \%)$ \\
Alien limb, n & $0(0 \%)$ \\
Myoclonus, $\mathrm{n}$ & $0(0 \%)$ \\
Tremor, n & $1(6 \%)$ \\
Hyperreflexia, $\mathrm{n}$ & $7(44 \%)$ \\
Babinski’s sign, $\mathrm{n}$ & $5(31 \%)$ \\
Supranuclear palsy, $\mathrm{n}$ & $16(100 \%)$ \\
Axial dystonia or rigidity, $\mathrm{n}$ & $16(100 \%)$ \\
Pseudobulbar palsy, $\mathrm{n}$ & $14(88 \%)$ \\
\end{tabular}

\section{Patients and Methods}

\section{Patient Selection}

In this study, we included 16 probable PSP patients [5 females and 11 males; age: 64.9 (6.4) years; Mini-Mental State Examination (MMSE) score: 21.0 (4.4)] and 20 cognitively normal controls [8 females and 12 males; age: 64.8 (6.4) years; MMSE score: 29.8 (0.6)]. We selected subjects retrospectively from the Dementia Registry, a database of our institute. These patients had been admitted to the infirmary at our institution between April 1996 and March 2002 to undergo an evaluation for dementia and had agreed to be registered in the database; they were followed up for $>2$ years. In order to ensure that the imaging protocols and the types of PET and MRI scanners were the same throughout the observation period, we performed the analyses with the instruments used initially.

The normal controls were matched for sex and age. The institutional ethics board approved this study. The participants' informed consents were obtained when they registered in the Dementia Registry database, but due to the retrospective design of this study informed consent was not required.

The MMSE scores of the participants were calculated. All patients were examined by neurologists and psychiatrists and underwent standard neurological and neuropsychological examinations, laboratory tests, electroencephalography, cranial MRI, and ${ }^{18}$ F-FDG-PET. The criteria for PSP of the National Institute of Neurological Disorders and Stroke Society were used to select patients for inclusion: a gradually progressive disorder; onset at $\geq 40$ years of age; vertical (upward and downward gaze) supranuclear palsy and prominent postural instability with falls in the 1st year of the disease, and no evidence of other diseases that could explain the features above. Our patient group had mild cognitive impairment [MMSE score: $21.0(4.4)]$.

All patients fulfilled the criteria for probable PSP, and all subjects who were included had been followed up for $\geq 3$ years after study entry. The diagnosis of PSP was confirmed in all cases at the follow-up evaluation. The patients' characteristics', including extrapyramidal signs, parkinsonism, and cortical symptoms, are shown in table 1 . All control subjects had MMSE scores $\geq 28$ and exhibited no clinical evidence of cognitive deficits or neurological disease. We did not detect any abnormal findings with regard to age-related atrophy or changes in white matter, including white matter hyperintensities. 


\section{Magnetic Resonance Imaging}

A 1.5-tesla Signa Horizon MRI system was used for this study (GE Medical Systems, Milwaukee, Wisc., USA). Sagittal, coronal, and axial $\mathrm{T}_{1}$-weighted spin echo images (TR/TE/ NEX: 550/15/2; $5 \mathrm{~mm}$ thick, $2.5-\mathrm{mm}$ gap) and axial $\mathrm{T}_{2}$-weighted fast spin echo images $(3,000 / 21,105 / 2)$ were obtained to detect abnormalities. VBM analyses were conducted using coronal 3D spoiled gradient echo (SPGR) images [23, 24](14/3/2, $20^{\circ}$ flip angle, $220-\mathrm{mm}$ field of view, $256 \times 256$ matrix, $124 \times 1.5 \mathrm{~mm}$ contiguous sections). The 3D-SPGR images were constructed with a voxel size of $0.86 \times 0.86 \times 1.5 \mathrm{~mm}$. Then, the images were reconstructed into $2.0-\mathrm{mm}$ isotropic voxel images by the SPM/VBM methods.

\section{Positron Emission Tomography}

The detailed PET procedure has been described previously [15]. In brief, FDG-PET images were obtained using a Headtome IV scanner (Shimadzu, Kyoto, Japan). All subjects fasted for at least $4 \mathrm{~h}$ before the PET scan. The subjects were examined in the resting condition with their eyes closed and ears unplugged. After a transmission scan, a 12-min emission scan was started $60 \mathrm{~min}$ after an intravenous injection of $185-370 \mathrm{MBq}$ FDG. The data were collected in $128 \times 128$ matrices. The slice number was 14 , and the slice interval was $6.5 \mathrm{~mm}$ in the z-motion mode.

\section{Data Analysis}

Anatomical normalization and statistical processing of the PET and MR images were performed using SPM for Windows, version 8 (SPM 8; Wellcome Department of Cognitive Neurology, London, UK). The calculations and image matrix manipulations were performed using MATLAB R2009b (MathWorks, Natick, Mass., USA).

For the VBM analysis using SPM 8, all coronal SPGR MRI datasets were reconstructed into axial datasets and subsequently converted to ANALYZE format and displayed with the right hemisphere on the right side of the image.

The MRI data were analyzed using VBM, implemented in SPM 8. First, conventional VBM was performed using the default template. Next, we applied DARTEL (an algorithm for accurate diffeomorphic image registration), implemented as a toolbox in SPM 8, to create a set of group-specific templates. Using these templates, the brain images were segmented, normalized, and modulated. The output images remained in the average brain space. Additional warping from the Montreal Neurological Institute space was provided to the brain images. Finally, the gray matter probability values were smoothed using an 8-mm full-width at half maximum Gaussian kernel to match the PET analysis. The DARTEL procedure, in addition to the previous spatial normalization methods that were developed by Ashburner [25], improves anatomical precision [26].

For FDG-PET, all individual PET images were co-registered with MRI. The images were transformed into a standard stereotactic anatomical space using the DARTEL flow that was created in the MRI normalization. Further, all PET and MRI images were smoothed using an isotropic 8-mm Gaussian kernel to increase the signal-to-noise ratio and compensate for differences in gyral anatomy between individuals. The individual FDG images were adjusted by proportional scaling to a mean value of $5.0 \mathrm{mg} 100 \mathrm{ml}^{-1} \mathrm{~min}^{-1}$.

The metabolic activity, gray matter, and white matter were compared between patients and controls and analyzed by a 2-sample $t$ test. The correlations between MMSE scores and brain structures were then examined with regard to metabolic activity, gray matter, and white matter by voxel-wise regression analysis, considering total intracranial volume, gender, and age as nuisance parameters.

Each comparison between patients and controls was analyzed by a statistical design in the 2-sample $t$ test. In this study, we applied an uncorrected threshold of $p<0.001$ on the 

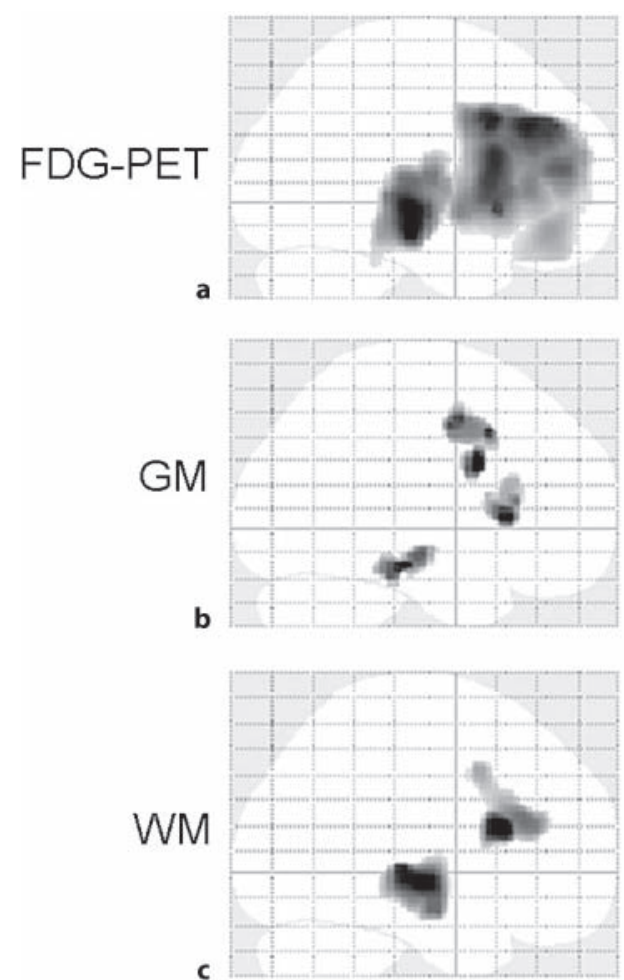
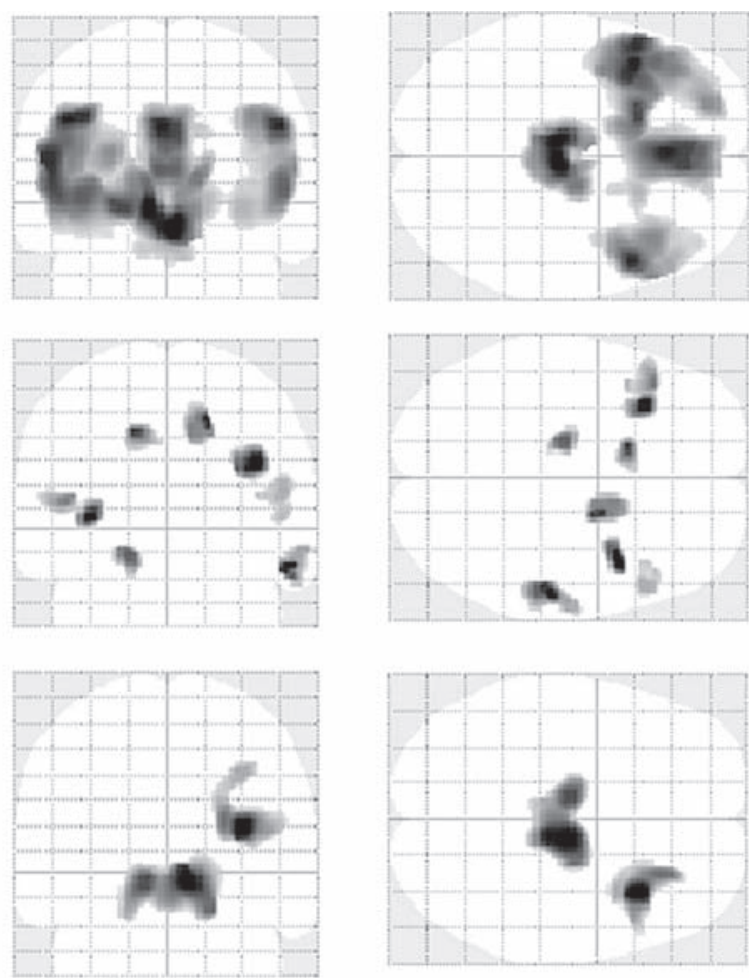

Fig. 1. SPM in patients and age-matched healthy controls. a Areas showing significantly lower hypometabolism in PSP patients are highlighted $(\mathrm{p}<0.001$, uncorrected). PET analysis of metabolic reductions in bilateral frontal lobes and midbrain tegmentum in the PSP group and normal controls. $\mathbf{b}$ Areas showing significant reductions in gray matter (GM) in PSP patients are highlighted ( $p<0.001$, uncorrected). Gray matter volume in the bilateral frontal lobes, anterior cingulate gyrus, right middle temporal gyrus, and right midbrain in PSP patients and normal controls. c Areas showing significant reductions in white matter $(\mathrm{WM})$ in PSP patients are highlighted $(\mathrm{p}<0.001$, uncorrected). White matter volume in the bilateral thalami, midbrain tegmentum, and right frontal lobes.

basis of the results obtained in the previous studies, in which the frontal lobes and the midbrain were found as the regions of hypometabolism [15-18] or decreased gray matter [12, 13] in patients with PSP.

\section{Results}

Comparison with Normal Controls

Using FDG-PET, we observed a reduction in metabolic activity in the bilateral frontal lobes and midbrain tegmentum in the PSP group compared with normal controls $(\mathrm{p}<0.001$, uncorrected; fig. 1a).

In the MRI VBM study, we compared gray matter volume in the bilateral frontal lobes, anterior cingulate gyrus, right middle temporal gyrus, and right midbrain between patients and normal controls ( $\mathrm{p}<0.001$, uncorrected; fig. $1 \mathrm{~b}$; table 2$)$. White matter volume decreased significantly in the bilateral thalami, midbrain tegmentum, and right frontal lobes ( $\mathrm{p}<$ 0.001 , uncorrected; fig. $1 c$; table 2). The decrease in white matter in the midbrain was more marked than the reduction in gray matter in the visual analysis. 
Table 2. Regions showing significant differences between PSP patients and controls

\begin{tabular}{|c|c|c|c|c|c|c|}
\hline \multirow[t]{2}{*}{ Comparison } & \multirow[t]{2}{*}{ Brain region } & \multicolumn{4}{|c|}{ Talairach coordinates } & \multirow[t]{2}{*}{ t value } \\
\hline & & side & $\mathrm{x}$ & $\mathrm{y}$ & $\mathrm{z}$ & \\
\hline \multirow[t]{5}{*}{ FDG-PET: PSP < NC } & Thalamus & $\mathrm{R}$ & 8 & -22 & 12 & 7.12 \\
\hline & Middle frontal gyrus & $\mathrm{L}$ & -38 & 54 & 8 & 6.71 \\
\hline & Lateral frontal lobe & $\mathrm{L}$ & -50 & 16 & 3 & 5.07 \\
\hline & Lateral frontal lobe & $\mathrm{R}$ & 54 & 17 & -4 & 6.60 \\
\hline & Medial frontal lobe & $\mathrm{R}$ & 6 & 40 & 24 & 6.02 \\
\hline \multirow[t]{7}{*}{ Gray matter: $\mathrm{PSP}<\mathrm{NC}$} & Lateral frontal lobe & $\mathrm{R}$ & 38 & 9 & 29 & 5.46 \\
\hline & Inferior frontal gyrus & $\mathrm{R}$ & 49 & 22 & 19 & 3.60 \\
\hline & Middle temporal gyrus & $\mathrm{R}$ & 53 & -25 & -8 & 5.26 \\
\hline & Anterior cingulate gyrus & $\mathrm{R}$ & 17 & 4 & 44 & 4.95 \\
\hline & Midbrain & $\mathrm{L}$ & -16 & -16 & -5 & 4.12 \\
\hline & Lateral frontal lobe & $\mathrm{L}$ & -34 & 20 & 8 & 5.22 \\
\hline & Anterior cingulate gyrus & $\mathrm{L}$ & -13 & 15 & 39 & 4.22 \\
\hline \multirow[t]{5}{*}{ White matter: $\mathrm{PSP}<\mathrm{NC}$} & Thalamus & $\mathrm{R}$ & 8 & -16 & 6 & 6.14 \\
\hline & Thalamus & $\mathrm{L}$ & -8 & -15 & 1 & 5.58 \\
\hline & Lateral frontal lobe & $\mathrm{R}$ & 34 & 27 & 19 & 6.24 \\
\hline & Midbrain & $\mathrm{L}$ & -4 & -18 & -5 & 4.32 \\
\hline & Midbrain & $\mathrm{R}$ & 12 & -15 & -9 & 4.14 \\
\hline
\end{tabular}

Table 3. Regions that correlate with MMSE score in PSP patients

\begin{tabular}{lllllll}
\hline & Brain region & \multicolumn{2}{l}{ Talairach coordinates } & \multirow{2}{*}{ t value } \\
\cline { 3 - 5 } & & side & $\mathrm{x}$ & $\mathrm{y}$ & $\mathrm{z}$ & \\
\hline \multirow{2}{*}{ FDG-PET } & Middle frontal gyrus & $\mathrm{L}$ & -38 & 35 & 26 & 4.77 \\
Gray matter & Anterior cingulate gyrus & $\mathrm{L}$ & -6 & 25 & 27 & 5.36 \\
& Rectal gyrus & $\mathrm{L}$ & -5 & 27 & 15 & 4.43 \\
& Rectal gyrus & $\mathrm{R}$ & 9 & 24 & -16 & 4.37 \\
& Middle frontal gyrus & $\mathrm{L}$ & -36 & 48 & 12 & 4.56 \\
\multirow{2}{*}{ White matter } & Inferior frontal gyrus & $\mathrm{L}$ & -37 & 35 & 3 & 6.97 \\
& Middle frontal gyrus & $\mathrm{L}$ & -23 & 39 & 20 & 5.24 \\
\hline
\end{tabular}

The extent to which the white matter decreased was less than the decrease in hypometabolism in PSP patients. The decreases in gray and white matter in the midbrain tegmentum were comparable.

\section{Metabolic and Structural Correlations with MMSE}

A positive metabolic and structural correlation with MMSE was observed in the left frontal lobe in regression analysis ( $\mathrm{p}<0.001$, uncorrected; fig. 2; table 3). Additional reductions in gray matter were noted in the right frontal lobe and bilateral rectal gyri (fig. 2; table 3).

\section{Representative Cases}

Figure 3 shows MR (fig. 3a) and FDG-PET (fig. 3b) images of a male patient with PSP (MMSE score: 23) and a normal female subject (MMSE score: 30 ) of the same age (68 years). Slight midbrain and bilateral frontal lobe atrophy was observed on visual inspection of the conventional $\mathrm{T}_{1}$-weighted image of the PSP patient compared with the healthy control 


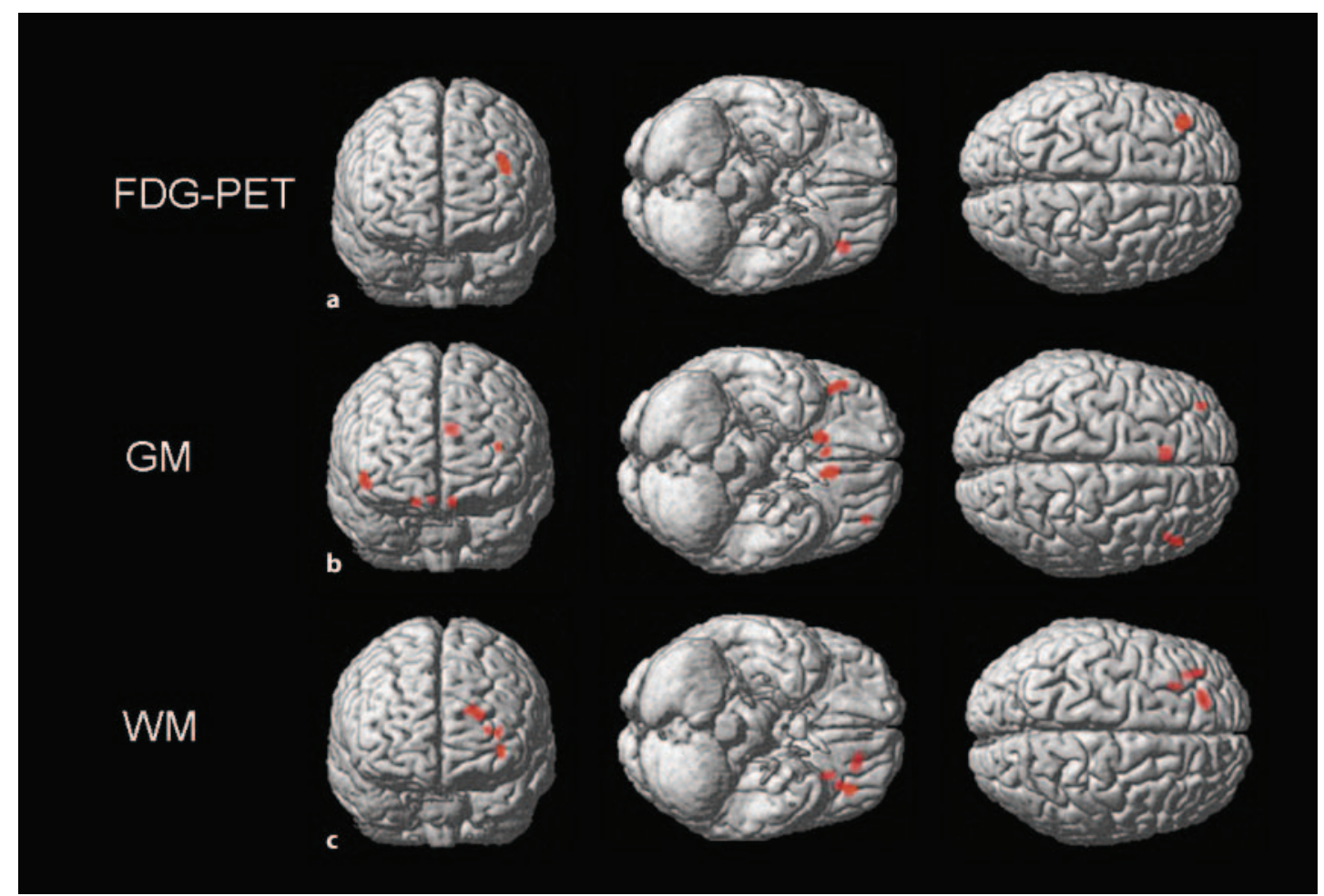

Fig. 2. Areas of significant positive correlations with MMSE scores in patients with PSP ( $p<0.001$, uncorrected). Correlations with metabolism (a), gray matter (GM) volume (b), and white matter (WM) volume (c). The left frontal lobe is highlighted in all analyses. Additional gray matter reduction is detected in the bilateral rectal gyri.

(fig. 3a); however, the PSP patient experienced more severe hypometabolism in the midbrain and bilateral frontal lobes, including the medial and lateral frontal lobes.

Figure 4 shows the MR (fig. 4a) and FDG-PET (fig. 4b) images of a demented male patient with PSP (MMSE score: 17) and a normal female subject (MMSE score: 30 ) of the same age (70 years). Abnormalities in the midbrain were not observed on visual inspection of the conventional $\mathrm{T}_{1}$-weighted image and FDG-PET images of the PSP patient compared with the healthy control; however, slight atrophy and more severe hypometabolism were noted in the PSP patient in the bilateral frontal lobes (fig. 4).

\section{Discussion}

This study is the first head-to-head comparison of FDG and VBM in the same group of PSP subjects. The FDG-PET and MRI scans showed that relatively similar regions experienced significant reductions in metabolic activity, gray matter, and white matter - primarily the frontal lobe and midbrain tegmentum. First, the visual extents of the regions in which there were significant changes were much greater on FDG-PET than on MRI. Further, the correlation between the frontal lobe and MMSE scores in PSP patients was similar in all evaluations. The midbrain tegmentum was not linked to the MMSE score in any evaluation. 


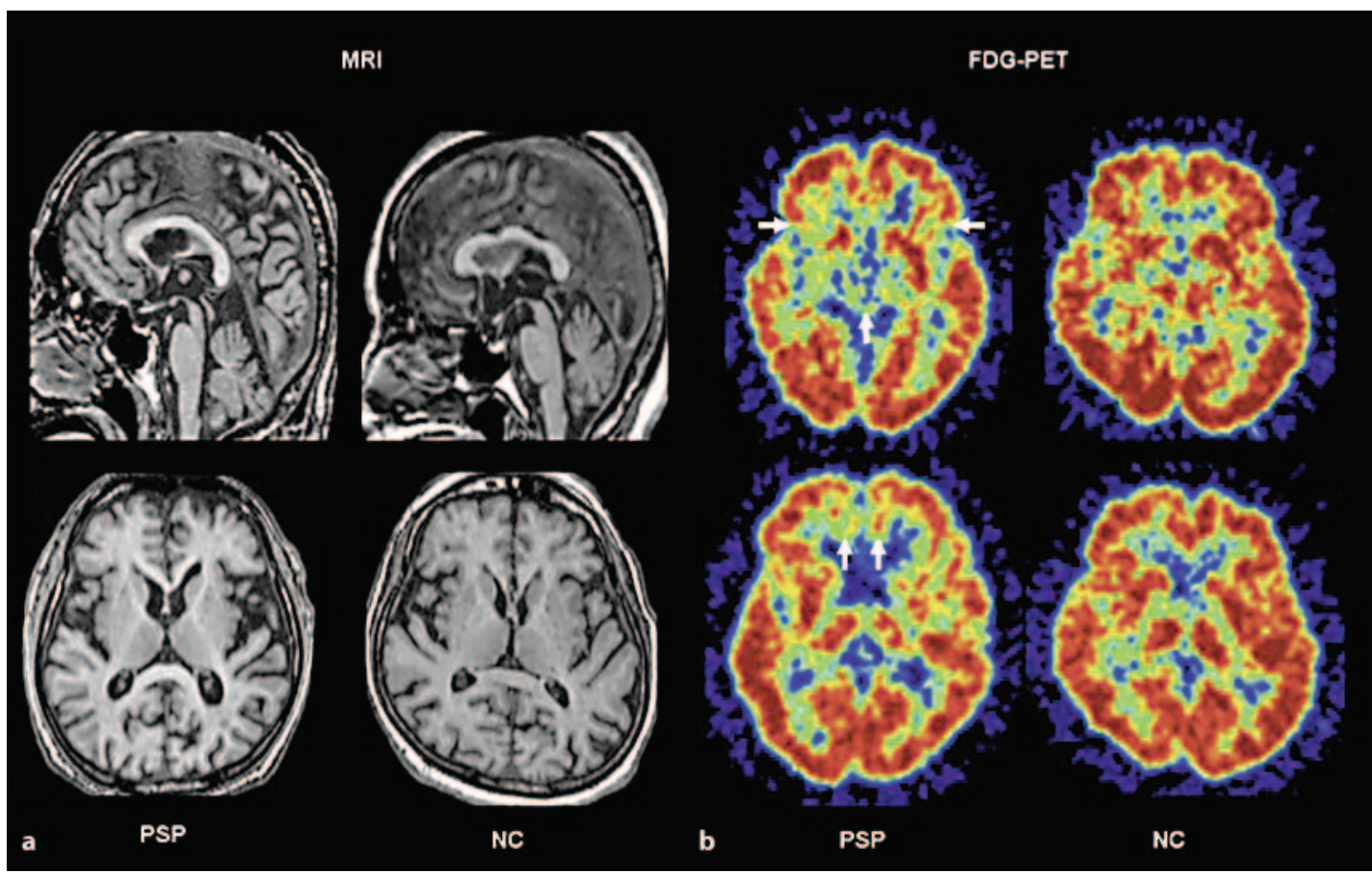

Fig. 3. Comparisons between the MRI (a) and FDG-PET (b) images of a 68-year-old male patient with PSP $($ MMSE score $=23)$ and a normal age-matched female control (NC; MMSE score = 30): slight atrophy in the midbrain and bilateral frontal lobes (a). More severe hypometabolism in the midbrain and bilateral frontal lobes, including the medial and lateral frontal lobes (b). The white arrows indicate the suspected abnormalities.

\section{Comparison between Cortical Atrophy and Hypometabolism in PSP Patients}

Compared with atrophy, the cluster volume of gray matter hypometabolism was greater, which implies that FDG-PET is more important in the differential diagnosis of PSP.

FDG-PET revealed hypometabolism in the bilateral frontal lobes and midbrain tegmentum, in agreement with previous PET studies [14-18].

VBM analysis disclosed significant reductions in gray matter in the lateral frontal lobe, including the middle and inferior frontal gyri, midbrain, and middle frontal gyrus. These results are consistent with previous VBM studies $[12,13]$. Additional gray matter reductions in the hippocampus and parahippocampal gyrus were noted in another study [12]. The cluster volume in our study appeared to be smaller than in previous VBM studies.

The problem in previous VBM studies has been the misregistration of structures [27]. Such misregistrations could be observed in the periventricular area and the deep brain structures, such as the striatal nucleus and brainstem [27]. The DARTEL method was quite useful to reduce the registration errors in these areas [26]. The greater reduction in midbrain gray matter (compared with white matter) that was observed in one study [12] might be explained by the misregistration of structures.

However, there would be some space to discuss the structural changes in the midbrain. In this study, $\mathrm{T}_{1}$-weighted imaging was used for VBM analysis. The brainstem has many nuclei with iron deposition. $\mathrm{T}_{1}$-weighted imaging is less sensitive to changes in iron deposits in the brainstem, which complicates the discrimination of MR signals between gray matter and white matter in the brainstem. This would directly affect the segmentation process in VBM. Another study that used surface rendering without segmenting the gray and white matter 


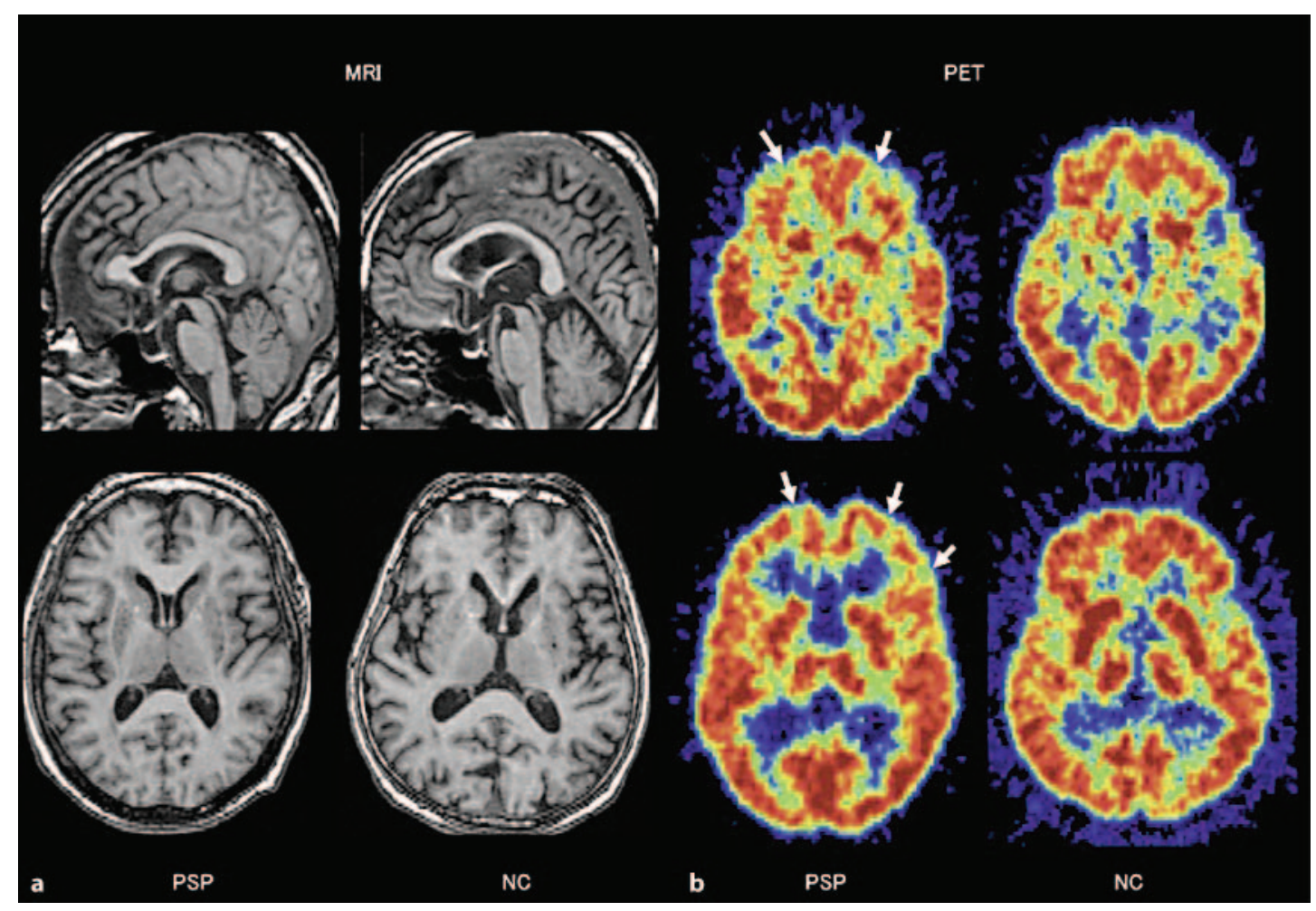

Fig. 4. MR (a) and FDG-PET (b) images of a demented male patient with PSP (MMSE score: 17) and a normal age-matched female control (NC; MMSE score: 30; 70 years). The white arrows indicate the suspected abnormalities in the frontal lobes.

observed brainstem atrophy in PSP patients [28]; however, due to limitations in measuring the volumes of regional structures, a new method for measuring brainstem atrophy in PSP should be developed

Our procedure - VBM using DARTEL - revealed greater reductions in the white than in the gray matter in the midbrain tegmentum. Further, the cluster volume of gray matter hypometabolism was much higher compared with gray matter atrophy in PSP patients.

\section{Brain Structures Correlate with Cognitive Impairment in PSP Patients}

We are the first to correlate brain structures and cognitive impairment in PSP. The frontal lobe was detected in all regression analyses. Our results show that atrophy and hypometabolism in the frontal lobe are as important as in the basal midbrain in the diagnosis of PSP patients exhibiting cognitive dysfunction. In fact, as shown in figure 4, we identified a demented PSP patient who only experienced hypometabolism and atrophy in the frontal lobe but not in the basal midbrain. If we consider PSP with regard to dementia, disease progression in the frontal lobe versus the midbrain is not synchronized.

The cognitive changes that occur in PSP are referred to as subcortical dementia, which is associated with cognitive impairment, including prominent deficits in recall and moderate forgetfulness, despite intact short-term and implicit perceptual memory [19]. These symptoms are similar to those in patients with frontal lobe dysfunction. Neuropsychological studies employing the Frontal Behavioral Inventory suggest the presence of frontal lobe dysfunction in PSP patients [29]. 
Moreover, neuropathological studies have demonstrated that the middle frontal gyrus is a representative region in which abnormal glial cells, accompanied by abnormal tau protein, appear [6], further supporting our data.

Notably, there were no significant findings in the midbrain. We speculate that parkinsonism correlates with changes in the midbrain tegmentum in PSP. The relationship between the Unified Parkinson's Disease Rating Scale score and structural changes has to be elucidated in future studies.

Our results suggest that in addition to the basal midbrain, the frontal lobe is a part not to be missed in the diagnosis of demented PSP patients who do not experience hypometabolism or atrophy in the midbrain.

A limitation of this study is that the diagnosis of PSP in our patients was not confirmed by autopsy. Postmortem examinations might have confirmed the accuracy of our results. Second, we did not compare the diagnostic performance between MRI and PET in diagnosing PSP. The conclusion of this study is restricted to the visual inspection.

Third, this study used an old type of PET scanner and the slice interval of $6.5 \mathrm{~mm}$ may be relatively large to evaluate midbrain metabolism precisely. Further, we compared the PET and MRI findings only with raw data and did not perform the atrophy correction. Atrophy may have contributed to the extent of the reduced signal shown in the PET scan. However, the methods for atrophy correction are still in the developing stages, and future studies have to develop an appropriate technique for atrophy correction. Finally, the use of VBM with the DARTEL algorithm continues to be refined; advances in its precision and accuracy might pave the way for a more detailed study of PSP pathology.

\section{Conclusions}

This head-to-head study of FDG-PET and MRI demonstrates that in PSP patients the extent of metabolic changes is greater than that of changes in gray and white matter. Early FDG-PET scanning is recommended for diagnosing PSP. Moreover, MMSE scores were associated with changes in the frontal lobe in PSP patients, which will be beneficial in diagnosing PSP patients with cognitive impairment who do not develop hypometabolism or atrophy in the midbrain and will contribute to future neuropathological and neurophysiological studies of PSP.

\section{Disclosure Statement}

The authors have no conflict of interest to report.

\section{References}

-1 Litvan I, Agid Y, Calne D, Campbell G, Dubois B, Duvoisin RC, Goetz CG, Golbe LI, Grafman J, Growdon JH, Hallett M, Jankovic J, Quinn NP, Tolosa E, Zee DS: Clinical research criteria for the diagnosis of progressive supranuclear palsy (Steele-Richardson-Olszewski syndrome): report of the NINDS-SPSP international workshop. Neurology 1996;47:1-9.

-2 Rehman HU: Progressive supranuclear palsy. Postgrad Med J 2000;76:333-336.

-3 Steele JC, Richardson JC, Olszewski J: Progressive supranuclear palsy. A heterogeneous degeneration involving the brain stem, basal ganglia and cerebellum with vertical gaze and pseudobulbar palsy, nuchal dystonia and dementia. Arch Neurol 1964;10:333-359. 
-4 Nath U, Burn DJ: The epidemiology of progressive supranuclear palsy (Steele-Richardson-Olszewski syndrome). Parkinsonism Relat Disord 2000;6:145-153.

-5 Komori T, Arai N, Oda M, Nakayama H, Mori H, Yagishita S, Takahashi T, Amano N, Murayama S, Murakami S, Shibata N, Kobayashi M, Sasaki S, Iwata M: Astrocytic plaques and tufts of abnormal fibers do not coexist in corticobasal degeneration and progressive supranuclear palsy. Acta Neuropathol 1998;96:401-408.

-6 Matsusaka H, Ikeda K, Akiyama H, Arai T, Inoue M, Yagishita S: Astrocytic pathology in progressive supranuclear palsy: significance for neuropathological diagnosis. Acta Neuropathol 1998;96:248252.

-7 Tedeschi G, Litvan I, Bonavita S, Bertolino A, Lundbom N, Patronas NJ, Hallett M: Proton magnetic resonance spectroscopic imaging in progressive supranuclear palsy, Parkinson's disease and corticobasal degeneration. Brain 1997;120(Pt 9):1541-1552.

8 Soliveri P, Monza D, Paridi D, Radice D, Grisoli M, Testa D, Savoiardo M, Girotti F: Cognitive and magnetic resonance imaging aspects of corticobasal degeneration and progressive supranuclear palsy. Neurology 1999;53:502-507.

-9 Schrag A, Good CD, Miszkiel K, Morris HR, Mathias CJ, Lees AJ, Quinn NP: Differentiation of atypical parkinsonian syndromes with routine MRI. Neurology 2000;54:697-702.

-10 Cordato NJ, Halliday GM, Harding AJ, Hely MA, Morris JG: Regional brain atrophy in progressive supranuclear palsy and Lewy body disease. Ann Neurol 2000;47:718-728.

-11 Oba H, Yagishita A, Terada H, Barkovich AJ, Kutomi K, Yamauchi T, Furui S, Shimizu T, Uchigata M, Matsumura K, Sonoo M, Sakai M, Takada K, Harasawa A, Takeshita K, Kohtake H, Tanaka H, Suzuki S: New and reliable MRI diagnosis for progressive supranuclear palsy. Neurology 2005;64: 2050-2055.

- 12 Padovani A, Borroni B, Brambati SM, Agosti C, Broli M, Alonso R, Scifo P, Bellelli G, Alberici A, Gasparotti R, Perani D: Diffusion tensor imaging and voxel based morphometry study in early progressive supranuclear palsy. J Neurol Neurosurg Psychiatry 2006;77:457-463.

-13 Brenneis C, Seppi K, Schocke M, Benke T, Wenning GK, Poewe W: Voxel based morphometry reveals a distinct pattern of frontal atrophy in progressive supranuclear palsy. J Neurol Neurosurg Psychiatry 2004; 75:246-249.

-14 Teune LK, Bartels AL, de Jong BM, Willemsen AT, Eshuis SA, de Vries JJ, van Oostrom JC, Leenders KL: Typical cerebral metabolic patterns in neurodegenerative brain diseases. Mov Disord 2010;25: 2395-2404.

-15 Juh R, Pae CU, Kim TS, Lee CU, Choe B, Suh T: Cerebral glucose metabolism in corticobasal degeneration comparison with progressive supranuclear palsy using statistical mapping analysis. Neurosci Lett 2005;383:22-27.

-16 Juh R, Kim J, Moon D, Choe B, Suh T: Different metabolic patterns analysis of parkinsonism on the ${ }^{18}$ F-FDG PET. Eur J Radiol 2004;51:223-233.

-17 Hosaka K, Ishii K, Sakamoto S, Mori T, Sasaki M, Hirono N, Mori E: Voxel-based comparison of regional cerebral glucose metabolism between PSP and corticobasal degeneration. J Neurol Sci 2002; 199:67-71.

-18 Garraux G, Salmon E, Peigneux P, Kreisler A, Degueldre C, Lemaire C, Destee A, Franck G: Voxelbased distribution of metabolic impairment in corticobasal degeneration. Mov Disord 2000;15:894904.

-19 Litvan I: Cognitive disturbances in progressive supranuclear palsy. J Neural Transm Suppl 1994;42: 69-78.

-20 Albert ML, Feldman RG, Willis AL: The 'subcortical dementia' of progressive supranuclear palsy. J Neurol Neurosurg Psychiatry 1974;37:121-130.

-21 Kertesz A, McMonagle P: Behavior and cognition in corticobasal degeneration and progressive supranuclear palsy. J Neurol Sci 2010;289:138-143.

-22 O'Keeffe FM, Murray B, Coen RF, Dockree PM, Bellgrove MA, Garavan H, Lynch T, Robertson IH: Loss of insight in frontotemporal dementia, corticobasal degeneration and progressive supranuclear palsy. Brain 2007;130:753-764.

-23 Ishii K, Sasaki H, Kono AK, Miyamoto N, Fukuda T, Mori E: Comparison of gray matter and metabolic reduction in mild Alzheimer's disease using FDG-PET and voxel-based morphometric MR studies. Eur J Nucl Med Mol Imaging 2005;32:959-963. 
-24 Kanda T, Ishii K, Uemura T, Miyamoto N, Yoshikawa T, Kono AK, Mori E: Comparison of grey matter and metabolic reductions in frontotemporal dementia using FDG-PET and voxel-based morphometric MR studies. Eur J Nucl Med Mol Imaging 2008;35:2227-2234.

-25 Ashburner J: A fast diffeomorphic image registration algorithm. Neuroimage 2007;38:95-113.

-26 Takahashi R, Ishii K, Miyamoto N, Yoshikawa T, Shimada K, Ohkawa S, Kakigi T, Yokoyama K: Measurement of gray and white matter atrophy in dementia with Lewy bodies using diffeomorphic anatomic registration through exponentiated Lie algebra: a comparison with conventional voxelbased morphometry. AJNR Am J Neuroradiol 2010;31:1873-1878.

-27 Bookstein FL: 'Voxel-based morphometry' should not be used with imperfectly registered images. Neuroimage 2001;14:1454-1462.

-28 Taki M, Ishii K, Fukuda T, Kojima Y, Mori E: Evaluation of cortical atrophy between progressive supranuclear palsy and corticobasal degeneration by hemispheric surface display of MR images. AJNR Am J Neuroradiol 2004;25:1709-1714.

-29 Borroni B, Alberici A, Agosti C, Cosseddu M, Padovani A: Pattern of behavioral disturbances in corticobasal degeneration syndrome and progressive supranuclear palsy. Int Psychogeriatr 2009;21: 463-468. 